\title{
Apo-Human Carbonic Anhydrase II Revisited: Implications of the Loss of a Metal in Protein Structure, Stability and Solvent
}

\section{Network,t,}

\author{
Balendu Sankara Avvaru§, Scott A. Busby\|, Michael J. Chalmers" , Patrick R. Griffin $\|$ \\ Balasubramanian Venkatakrishnan $\S$, Mavis Agbandje-McKenna§, David N. Silverman $\S, \pm,{ }^{*}$, \\ and Robert McKenna $\S^{*}$ \\ $\S$ Department of Biochemistry and Molecular Biology, Gainesville, FL 32610 \\ ${ }^{ \pm}$Department of Pharmacology and Therapeutics, and University of Florida, Gainesville, FL 32610 \\ "Department of Molecular Therapeutics, The Scripps Research Institute, Jupiter, FL 33458
}

\begin{abstract}
Human carbonic anhydrase II (HCA II) is a monomeric zinc-containing metalloenzyme that catalyzes the hydration of $\mathrm{CO}_{2}$ to form bicarbonate and a proton. The properties of the zinc have been extensively elucidated in catalysis, but less well studied as a contributor to structure and stability. Apo-HCA II (without zinc) was prepared and compared to holo-HCA II; in crystallographic structural features, in backbone amide H/D exchange, and in thermal stability. The removal of zinc from the active site has no effect on either the topological fold of the enzyme or the ordered water network in the active site. However, the removal of the zinc alters the collective electrostatics of the apo-HCA II that result in the following differences from that of the holoenzyme; 1 ) the main thermal unfolding transition of the apo-HCA II is lowered by $8{ }^{\circ} \mathrm{C}, 2$ ) the relative increase in thermal mobility of atoms of the apo-HCA II was not observed in the vicinity of the active site but manifested on the surface of the enzyme, and 3) the side chain of His 64, the proton shuttle residue that sits on the rim of the active site, is oriented outwards and is associated with additional ordered "external" waters, as opposed to a near equal inward and outward orientation in the holo-HCA II.
\end{abstract}

Carbonic anhydrases $(\mathrm{CAs})^{1}$ are ubiquitous enzymes found in all phyla of life and are intricately involved in many physiological processes. They catalyze the reversible hydration/ dehydration of carbon dioxide/bicarbonate (1). Of all the CA isoforms, the zinc metalloenzyme human carbonic anhydrase II (HCA II) is the most studied and probably the best understood. The reversible hydration of carbon dioxide to bicarbonate and a proton is best described as a ping-pong mechanism that is composed of two discrete steps $(2,3)$. The first step of hydration involves the formation of the zinc-bicarbonate complex through a nucleophilic attack on the substrate $\mathrm{CO}_{2}$ by the zinc-bound hydroxide (eq 1). The bicarbonate is then exchanged for a

\footnotetext{
$\dagger$ This work was supported by a grant from the NIH GM 25154. Hydrogen/deuterium exchange analysis was carried out by SAB, MJC and PRG who were supported by a grant from the NIH GM 084041.

¥Coordinates and structure factors have been deposited in the Protein Data Bank as entry 3GZ0.

*To whom correspondence should be addressed. R.M.: Department of Biochemistry and Molecular Biology, College of Medicine, University of Florida, Box 100245, Gainesville, Florida 32610; phone, (352) 392-5696; fax, (352) 392-3422; e-mail; rmckenna@ ufl.edu. D.N.S.: Department of Pharmacology, College of Medicine, University of Florida, Box 100267, Gainesville, Florida 32610; phone, (352) 392-3556; fax, (352) 392-9696; e-mail, silvrmn@ufl.edu..

SUPPORTING INFORMATION AVAILABLE

Experimental procedures and results of circular dichroism experimentation. Crystallographic and hydrogen/Deuterium exchange data. This material is available free of charge via the Internet at http://pubs.acs.org.
} 
water molecule forming the zinc-bound water (eq 1). The subsequent second step involves the regeneration of the zinc-bound hydroxide through the transfer of a proton from the zinc-bound water to the bulk solvent (eq 2). The intramolecular proton transfer is widely speculated to occur between the zinc-bound solvent and the side chain of His 64, that sits on the rim of the active site, through a network of ordered active site waters to an 'in' conformer of His 64 (pointing towards the active site) which then rotates to an 'out' conformer (pointing towards the bulk solvent) (Figure 1,2) (3-6).

\begin{tabular}{|c|c|c|c|c|}
\hline & & \multicolumn{3}{|c|}{$\mathrm{H}_{2} \mathrm{O}$} \\
\hline $\mathrm{CO}_{2}+\mathrm{EZnOH}^{-}$ & $\rightleftharpoons$ & $\mathrm{EZnHCO}_{3}{ }^{-}$ & $\rightleftharpoons$ & $\mathrm{EZnH}_{2} \mathrm{O}+\mathrm{HCO}_{3}{ }^{-}$ \\
\hline
\end{tabular}

The HCA II structure can best be described as a single domain mixed $\alpha / \beta$ globular protein. The central structural motif is a twisted $\beta$-sheet of 8 strands $(\beta \mathrm{A}-\mathrm{H})$, which is flanked by seven $\alpha$ helices $(\alpha \mathrm{A}-\mathrm{G})$. The catalytic active site is characterized by a conical cleft that is approximately $15 \AA$ deep with the zinc residing in the interior. The zinc is tetrahedrally coordinated by three histdine ligands (His 94, His 96, and His 119) and a bound water/hydroxyl. The zinc-ligand distances are all $\sim 2.1 \AA$ including the zinc-bound solvent molecule $(6,7)$.

It has been previously proposed by Cox et al., that there is a hierarchy of zinc ligands in the active site (that function as distinct shells of residues to stabilize the zinc ion) (8). The firstshell, or direct zinc ligands, are the three histidine residues His 94, His 96, His 119 and a solvent molecule. The second-shell, or indirect ligands, stabilize the direct ligands and help position them for zinc ion coordination. Residue Gln 92 stabilizes His 94, Glu 117 stabilizes His 119, and the backbone carbonyl oxygen of Asp 244 stabilizes His 96, while residue Thr 199 hydrogen bonds with the zinc-bound solvent. Finally a third-shell of stabilization was proposed of a cluster of aromatic residues (Phe 93, Phe 95, and Trp 97) that anchor the $\beta$-strand $\beta F$ that contains His 94 and His 96 (Figure 1) (8).

The properties of the zinc in regards to catalysis are well studied given its importance in the catalytic mechanism. However, its influence on the structural stability of the enzyme has not been thoroughly explored. The active site of apo-HCA II has high affinity for zinc with a value of $\mathrm{pK}_{\mathrm{D}}$ near 12 (9). Although tightly bound, the zinc is readily removed from HCA II by treatment with chelators and can be replaced by a number of other metals $(10,11)$. Håkansson et al. have previously reported the structure of apo-HCA II to $1.8 \AA$ resolution for crystals prepared at $\mathrm{pH} 7.0$ (12). They noted no significant differences in structure compared with holoHCA II. In fact, many of the ordered water molecules of the active site cavity were observed in the apo-HCA II as well, although the water molecule corresponding to the location of the zinc-bound solvent was shifted $\sim 0.8 \AA$ toward the unoccupied zinc site.

Since HCA II is monomeric, it serves as a good model for a structural stability study on the loss of metal from the active site, as the stability of the enzyme is not complicated by multimeric subunit interactions as observed in other protein structures. Furthermore, the apo- and holoHCA II crystallize under similar conditions and were isomorphous in their unit cells. Hence this study revisits the study of apo-HCA II, and examines the effects of the removal of active site zinc on the structure and stability of the enzyme.

This work has extended examination of the structure of apo-HCA II to $1.26 \AA$ A resolution, to that previously reported by Håkansson et al. (12), providing better defined placement of the ordered water molecules and allowing a detailed structural correlation to the recently 
determined 1.1 A resolution holo-HCA II (6). Of note was the conformation of the side chain of His 64 that differs in the apo- compared to holo-HCA II. In the holo-HCA II, His 64 appears about equally distributed in an inward and outward orientation (6), whereas in the apo-HCA II it is predominantly in the outward orientation. Also, the relative mobility of the apocompared with the holo-HCA II was made through the comparison of X-ray crystallography B factors and backbone amide H/D exchange measured by mass spectrometry. Interestingly, the increase in the thermal motion of the apo-HCA II, as indicated by crystallographic B factors and H/D exchange, was not observed in the immediate vicinity of the zinc binding site but rather on the surface of the enzyme. The net effect of this observed difference in thermal motion was experimentally measured in solution using calorimetry, and the melting temperature $\left(T_{\mathrm{m}}\right)$ of the apo-HCA II was shown to be $8{ }^{\circ} \mathrm{C}$ lower than holo-HCA II. Circular dichroism data also confirmed this value and demonstrated the initial melting event to be associated with the unfolding of the $\beta$-sheet structure followed by $\alpha$ - helix secondary structural elements. This data, taken as a whole, provides useful information of the contribution of the zinc to stability of carbonic anhydrase and builds on our general knowledge of metalloenzyme stability.

\section{MATERIALS AND METHODS}

\section{Expression and Purification of holo-HCA II}

The plasmid encoding HCA II was transformed into E.coli BL21 cells through standard procedures and the transformed cells were expressed at $37^{\circ} \mathrm{C}$ in LB medium containing 100 $\mu \mathrm{g} / \mathrm{ml}$ ampicillin (13). Holo-HCA II production was induced by the addition of isopropyl thiogalactoside to a final concentration of $1 \mathrm{mM}$ at an $\mathrm{O}^{\mathrm{D}} \mathrm{D}_{600}$ of $0.6 \mathrm{AU}$. The cells were harvested after $4 \mathrm{hrs}$ of post induction. The cell pellets were lysed and holo-HCA II was purified through affinity chromatography using pAMBS resin as has been described elsewhere (14).

\section{Preparation of Apo-HCA II}

The zinc was chelated through the incubation of the holo-HCA II at $20^{\circ} \mathrm{C}$ in the chelation buffer (100mM pyridine-2,6-dicarboxylic acid; $25 \mathrm{mM}$ MOPS; $\mathrm{pH} 7.0$ ) for $8 \mathrm{hrs}$. The enzyme was buffer exchanged against 50mM Tris; $\mathrm{pH} 7.8$ to remove the chelating agent (11). The loss of enzyme activity was verified through kinetic studies as has been described elsewhere (15). The enzyme activity was revived through the addition of $1 \mathrm{mM} \mathrm{ZnCl}_{2}$, attributing the loss of activity to the absence of zinc rather than to the denaturation of the enzyme.

\section{Crystallization and X-ray data collection of Apo-HCA II}

Crystals of apo-HCA II were obtained using the hanging drop vapor diffusion method (16). 10 $\mu l$ drops of equal amounts of protein and precipitant were equilibrated against precipitant solution (1.3 M sodium citrate; $100 \mathrm{mM}$ Tris- $\mathrm{HCl}$; $\mathrm{pH} 9.0)$ at room temperature $\left(\sim 20^{\circ} \mathrm{C}\right)(6)$. A crystal was cryoprotected by quick immersion into $20 \%$ glycerol precipitant solution and flash-cooled by exposing it to a gaseous stream of nitrogen at $100 \mathrm{~K}$. X-ray diffraction data were collected at the Cornell High Energy Synchrotron Source (CHESS) beamline A1 $\left(\lambda=0.9772 \AA\right.$ ), using the oscillation method with intervals of $1^{\circ}$ steps on an ADSC Quantum $210 \mathrm{CCD}$ detector, with a crystal to detector distance of $65 \mathrm{~mm}$. Indexing, integration, and scaling were performed using HKL2000 (17).

\section{Structure determination of Apo-HCA II}

The crystal structure of holo-HCA II (PDB accession code: 2CBA) (12) was used to obtain initial phases of the apo-structure using SHELXL (18). The zinc and all solvent molecules were removed to avoid model bias. 5\% of the unique reflections were selected randomly and excluded from the refinement data set for the purpose of $R_{\text {free }}$ calculations (19). Structural refinement proceeded using SHELXL initially with data from 20.0 to $2.0 \AA$ A resolution. The 
protein geometry was defined using the default constrains of conjugate-least squares (CGLS) mode in SHELXL. Each round of CGLS comprised of 15 cycles of refinement. $2 \mathrm{~F}_{\mathrm{o}}-\mathrm{F}_{\mathrm{c}}$ and $\mathrm{F}_{\mathrm{O}}-\mathrm{F}_{\mathrm{c}}$ electron density Fourier difference maps were calculated after each successive round of CGLS and manually inspected the graphics program Coot (20) for further fine-tuning of the model and the incorporation of solvent molecules. After some initial rounds of CGLS refinement, the resolution was extended to $1.26 \AA$ and subsequently after several more cycles of refinement and solvent addition the $\mathrm{R}_{\mathrm{work}}$ and $\mathrm{R}_{\text {free }}$ converged to 18.0 and 21.5 , respectively.

Based on the electron density maps, seven amino acids (Asp 34, His 36, Lys 39, Lys 112, Glu 117, Ser 217 and Glu 238) were built with dual conformations. These dual occupancy sidechains were incorporated into the model by splitting at the C $\alpha$ position. In the holo-HCA II structure these residues were Ile 22, Asp 34, His 64, Asp 175, Glu 187, Ser 217, and Ser 220 (6). It was noted only residues Asp 34 and Ser 217 exhibited dual conformers in both apo- and holo-HCA II. The rationale for these differences in residues could not be explained structurally.

The apo-HCA II model was further subjected to several cycles of full anisotropic refinement and hydrogen riding which led a convergence of $R_{\text {cryst }}$ and $R_{\text {free }}$ to 14.0 and 18.7, respectively. The geometry of the final model was checked using the PROCHECK algorithm (21). The RMSD for bond lengths and bond angles were found to be within accepted limits of $0.004 \AA$ and $1.4^{\circ}$, respectively. It was observed that $88 \%$ of the dihedral angles were in the most favored region while the rest were in the allowed region with the exception of $0.5 \%$ which were in the generously allowed region. The average $\mathrm{B}$ factors for the main- and side-chain atoms were determined to be 18.6 and $23.1 \AA^{2}$, respectively. The refined model included 250 water molecules with an average solvent isotropic B factor of $30.2 \AA^{2}$. The geometry and statistics of the final model are summarized in Tables 1 and $\mathrm{S} 1$.

\section{Hydrogen/Deuterium Exchange (HDX) Analysis}

Solution phase amide HDX was performed on apo- and holo-HCA II using a fully automated system that is described elsewhere $(22,23)$. Briefly, $4 \mathrm{~mL}$ of a $15 \mathrm{mM}$ protein solution containing either apo- or holo-HCA II ( $25 \mathrm{mM}$ Tris-Cl, $\mathrm{pH} 7.9,150 \mathrm{mM} \mathrm{KCl}, 2 \mathrm{mM}$ DTT) was diluted up to $20 \mathrm{~mL}$ with $\mathrm{D}_{2} \mathrm{O}$ dilution buffer (25mM Tris-Cl, $\mathrm{pH} 7.9,150 \mathrm{mM} \mathrm{KCl}, 2 \mathrm{mM}$ DTT) and incubated at $4^{\circ} \mathrm{C}$ for the following periods of time: $1,30,60,900,3600$ and $260000 \mathrm{~s}$. Following deuterium on-exchange, unwanted forward and back exchange was minimized and the protein was denatured by dilution to $50 \mathrm{ml}$ with $1 \%$ TFA in $3 \mathrm{M}$ urea (held at $1{ }^{\circ} \mathrm{C}$ ). The protein sample was then passed across an immobilized pepsin column (prepared in-house) at $200 \mathrm{ul} / \mathrm{min}(0.1 \%$ TFA, $1^{\circ} \mathrm{C}$ ) and the resulting peptides were trapped onto a $\mathrm{C}_{18}$ trap column (Microm Bioresources). Peptides were gradient eluted ( $4 \% \mathrm{CH} 3 \mathrm{CN}$ to $40 \% \mathrm{CH} 3 \mathrm{CN}, 0.3 \%$ formic acid over $15 \mathrm{~min}$ at $2{ }^{\circ} \mathrm{C}$ ) over a $2.1 \mathrm{~mm} \times 50 \mathrm{~mm} \mathrm{C}_{18}$ reverse phase HPLC column (Hypersil Gold, Thermo Electron) and electrosprayed directly into a linear ion trap mass spectrometer (LTQ, ThermoElectron). Data were processed using in-house software $(24,25)$ and Microsoft Excel followed by visualization with pyMol (DeLano Scientific, South San Francisco CA). Average percent deuterium incorporation was calculated for 35 regions of the holo- and apo-HCA II following 1, 30, 60, 900, 3600 and $260000 \mathrm{~s}$ of on-exchange with deuterium. To determine differences in exchange between apo- and holo-HCA II, the average percent deuterium values for all 35 regions of apo-HCA II from the average percent deuterium values of the same 35 regions of the holo-HCA II were subtracted (Table S2).

\section{Differential Scanning Calorimetry (DSC)}

DSC experiments were performed using VP-DSC calorimeter (Microcal, Inc., North Hampton, MA) with a cell volume of $\sim 0.5 \mathrm{ml}$. Apo- and holo-HCA II samples were buffered in $50 \mathrm{mM}$ Tris- $\mathrm{HCl}, \mathrm{pH} 7.0$ at protein concentrations of $4 \mu \mathrm{M}$ and $5 \mu \mathrm{M}$ respectively. DSC scans were collected from $30{ }^{\circ} \mathrm{C}$ to $90{ }^{\circ} \mathrm{C}$ with a scan rate of $90{ }^{\circ} \mathrm{C} / \mathrm{hr}$. The calorimetric enthalpies of 
unfolding were calculated by integrating the area under the peaks in the thermograms after adjusting the pre and post transition baselines. The thermograms were fit to a two state reversible unfolding model to obtain van't Hoff enthalpies of unfolding.

The melting temperatures $\left(T_{\mathrm{m}}\right)$ of apo- and holo-HCA II occurred at characteristic mid points on the DSC curves indicating a two state transition. The difference in Gibbs free energy ( $\Delta G$ ${ }^{\circ}$ ) at a given temperature $T$ was therefore calculated using the following equation (26).

$\Delta G^{\mathrm{O}} \quad(T)=\Delta H \quad{ }^{\circ} \mathrm{m} \quad\left(1-T / T_{\mathrm{m}}\right)+\Delta C_{p}\left[\begin{array}{llllll}(T & - & \left.T_{\mathrm{m}}\right) & - & T & \ln \left(T / T_{\mathrm{m}}\right)\end{array}\right]$

Here $\Delta H^{\circ} \mathrm{m}$ is the calorimetric enthalpy at $T_{\mathrm{m}}$ and $\Delta C_{p}$ is the observed change in heat capacity between the folded and unfolded states. The denaturation enthalpies $\left(\Delta H^{\circ}\right)$ and entropies $\left(\Delta S^{\circ}\right)$ were calculated for a given temperature T using the following Kirchhoff's law equations.

$$
\begin{aligned}
& \Delta H \quad 0 \quad(T)=\Delta H \quad 0 \quad \mathrm{~m}+\Delta C_{p}\left(T-T_{\mathrm{m}}\right) \\
& \Delta S \quad \circ \quad(T)=\Delta S \quad 0 \quad \mathrm{~m}+\Delta C_{p} \quad \ln \left(T / T_{\mathrm{m}}\right)
\end{aligned}
$$

\section{RESULTS}

\section{Residual electron density for metal ion}

We observed no significant differences in the overall fold between the apo- and holo-HCA II structures. However, the residual $\mathrm{F}_{\mathrm{o}}-\mathrm{F}_{\mathrm{c}}$ electron density Fourier difference map for apo-HCA II displayed some weak electron density at the presumed "removed" zinc site. Håkansson et $a l$. also reported a similar observation and attributed this to the presence of sodium ions in their buffers (12). In this study, the buffers also contained sodium ions and the current data are also consistent with this explanation, or possibly a water molecule, which could have filled the void space left by the removal of the zinc. An alternate, more consistent consideration is that this site contained residual zinc from glassware and solutions, despite care in avoiding this contamination, as apo-HCA II has an extremely high affinity for zinc (9). The apo-HCA II structure was refined assuming a partial zinc occupancy of $10 \%$ based on the relative B factor of the holo-HCA II structure at $1.2 \AA$. In retrospect, the crystallization reagents and cryoprotectent solutions ought to have been treated for removal of exogenous zinc prior to crystallization. However catalytic activity studies (data not shown) using ${ }^{18} \mathrm{O}$ exchange methods (15), estimated the solution sample of apo-HCA II to contain a near equal zinc contamination of $10 \%$ based on relative activity to holo-HCA II. The nature of the apo-HCA II in both the crystal and solution was therefore assumed to be similar.

\section{Active site of apo-HCA II}

We observed the same ordered water structure in the apo-HCA II as was observed in the activesite cavity of holo-HCA II. This was also reported by Håkansson et al. (12). In apo-HCA II the solvent molecule corresponding to the zinc bound solvent of the holo-HCA II was observed to be shifted $\sim 0.8 \AA$ towards the unoccupied zinc binding site. In the absence of zinc, this shift may be sterically or energetically favorable. However this observation may also be an artifact of the partial occupancy of zinc. The active site water network has been shown to be supported through a network of hydrogen bonding interactions from the zinc-bound solvent and the sidechains of residues (Thr 7, Asn 62, His 64 and Asn 67) that line the active site (6). Previous studies have shown that this active-site water network is very sensitive to the amino acid sidechain interactions and can therefore be readily disturbed upon mutation of one or more of these 
residues (27). As the structure of the apo-HCA II reveals no change in the positions of the water molecules in the active site, this would indicate that both the zinc and the zinc-bound solvent play little role in the formation and/or stability of the solvent network (Figure 2).

A comparison between the crystallographic B factors (an indication of the thermal mobility of an atom) of the apo- and holo-HCA II structures, showed, with the exception of W1, that the solvent had similar thermal parameters (Figure S1). The water molecule W1 in holo-HCA II is partly stabilized by a hydrogen bond with the zinc-bound solvent. Hence, in the absence of the zinc, it seems a likely explanation, the removal of this interaction would account for the increased thermal motion of W1 observed in apo-HCA II structure (Figure S1).

In the apo-HCA II, His 64 appears predominantly (solely within experimental observations) in an outward conformer stabilized through a $\pi$ stacking interaction with the indole ring of Trp 205. In addition, the apo-HCA II structure revealed three "new" water molecules, termed W4, W5 and W6, previously not observed in the holo-HCA II (Figure 2). The water molecule W4 located in the region that would otherwise be occupied by His 64 in an inward conformer (as observed in the structures of holo-HCA II) (6). The other two additional waters, W5 and W6, are within hydrogen bonding distance to the side chain nitrogens of His64 and are bulk solvent waters, outside the active site cavity (Figure 2).

\section{Analysis of B factors for backbone atoms}

The apo-HCA II structure was compared to the recently reported $1.05 \AA$ A resolution structure of holo-HCA II (PDB code 2ILI) (6). An average B factors for each residue, based on the O, $\mathrm{N}$, and $\mathrm{C}$ backbone atoms, in apo-HCA II was calculated and normalized to the overall average $B$ value of the holo-HCA II. The normalized average $B$ factors for each residue of both the structures are plotted in Figure 3. The regions indicated by the black bars labeled 1,2 and 3 in Figure 3 were those observed to have significantly larger B factors for apo- than for holo-HCA II.

Inspection of the B factors for the apo-HCA II showed that the nearly complete absence of the metal was not associated with significant thermal instability for the residues within the active site cleft, with the exception of Asn 67 (Figure S2). The histidine ligands of the zinc and secondary shell stabilizing amino acids showed no increase in thermal vibration in apocompared with holo-HCA II (Figure 3, dark and light green arrows). But this analysis is suggestive that the absence of the zinc effects thermal stability of residues 53-75, 146-185 and 220-240 on the protein surface. However, the rate of increase in radial thermal-variation as a function of distance from the zinc was no greater than that observed in the holo-HCA II (Figure S3). It should, however be noted, that crystal contacts may interfere with the B factor analysis, and these results may have been dampened by crystal packing events. Although, again this was not apparent when studying those amino acids on the surface of the enzyme that were involved in such contacts. In Figure 3, these residues are indicated by tick marks and show mean average $\mathrm{B}$ factor of $25.4 \AA^{2}$ while the mean average $\mathrm{B}$ factor of the remaining residues is $19.5 \AA^{2}$ indicating a mionor difference in crystal versus non-crystal contacts. Instead in apoHCA II, the removal of the zinc seems to have perturbed the stability of the hydrophobic $\beta$ sheet-core (residues 50-75), which in turn seems to affect the stability of the outer $\alpha$ helices. Such an interpretation also seems to explain the higher thermal vibration of Asn 67, which lies on one of the $\beta$-sheets of the hydrophobic core that is destabilized in the absence of the zinc (Figure S2).

\section{Differential scanning calorimetry}

The thermal unfolding transitions of apo- and holo-HCA II were studied by DSC. A major unfolding transition, the observed dominant peak, was seen for both holo- and apo-HCA II 
(Figure 4). The transitions were recognized as endothermic peaks, that were centered at the $T_{m}$, with the maximum heat capacity $\left(C_{p}\right)$ occurring at the $T_{m}$. The $T_{m}$ of holo- was observed at $59 \pm 0.5^{\circ} \mathrm{C}$ while that of the apo- HCA II was found nearly $8{ }^{\circ} \mathrm{C}$ lower at $51 \pm 0.5{ }^{\circ} \mathrm{C}$. These values were $\sim 2{ }^{\circ} \mathrm{C}$ lower than the $\mathrm{CD}$ values (most likely a consequence of different protein concentration and buffers) (Figure S4). The calorimetric ethalphy at $T_{\mathrm{m}}\left(\Delta H^{\circ} \mathrm{m}\right)$ was calculated by integrating the area under the unfolding peak, normalized to the protein molar concentration that yielded values of $280 \mathrm{kcal} \mathrm{mol}^{-1}$ and $250 \mathrm{kcal} \mathrm{mol}^{-1}$ for apo- and holo-HCA II, respectively.

Although the experimentally observed DSC curves of apo- and holo- HCA II were fit to a twostate reversible unfolding model to yield Van't Hoff enthalpies (Table 2), the DSC curve of apo-HCAII does not fit to a two state model as precisely as the holo-HCAII due to a secondary peak observed at $58{ }^{\circ} \mathrm{C}$ (Figure 4A). However, this could be explained by the $10 \%$ of the apoHCA II samples that retained zinc despite stringent efforts to remove it. The DSC curve of apo-HCA II when resolved into two distinct peaks in Figure 5B reveals the secondary peak (shown as green in Figure 4B) to coincides with holo-HCA II peak and indicates that the secondary peak could be attributed to a $10 \%$ zinc contamination in the apo-HCA II samples. These results are in agreement with the crystallographic studies showing residual density that could be account for by assuming $\sim 10 \%$ zinc occupancy and the kinetic analysis that also agreed with this interpretation. As $\Delta G^{\circ}$ at $T_{m}$ in a two state reversible model is zero, a reference temperature of $55^{\circ} \mathrm{C}$, between the melting temperatures of apo- and holo-HCAII, was chosen

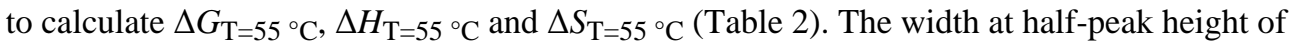
holo- was nearly a unit lower than apo-HCA II indicating a relatively higher cooperative transition between the native and denatured states in the holoenzyme. These results were confirmed using circular dichorism spectroscopy as an alternate technique (Figure S4).

\section{Backbone amide H/D exchange}

To understand the effects of the loss of zinc on the dynamics of HCA II, comprehensive differential, backbone amide HDX experiments were performed on both holo- and apo-HCA II. Exchange kinetics were measured for 35 regions of the enzyme and for each region, comparisons were made between the exchange kinetics of the holo- and apo-HCA II (Figure 5A). The results of these comparisons are given in Table S2 and Figure S5 with the percent change in deuterium incorporation of the apo- as compared to the holo-HCA II being reported for all 35 peptides. As was noted in the crystallographic B factor comparison, little changes in dynamics were observed when comparing holo- and apo-HCA II in regions containing the zinc binding sites (His 94, His 96 and His 119) or the cluster of hydrophobic amino acids (namely Val 121, Val 143, Leu 198, Thr 199, Val 207 and Trp 209) that comprise the hydrophobic environment of the active site.

However, significant differences were observed in peptides 58-66 that contain important hydrophilic residues of the active-site cavity such as Asn 62 and the proton shuttling residue His 64 . In this case, the amide exchange kinetics for this residue were significantly reduced in holo-compared with apo-HCA II suggesting a loss of stabilization of this region upon loss of zinc. Moreover, significant differences were observed in the amide exchange kinetics of two other regions of the enzyme, residues 147-189 and 224-239 (Figures 3 and 5). Peptides within these regions demonstrated reduced amide exchange kinetics in the holo- compared to the apoHCA II suggesting an increase in dynamics in these regions of the apo-HCA II which is also consistent with the crystallographic B factor analysis. Taken together, the HDX data corroborates the findings that the loss of zinc does not affect the dynamics of the metal binding site or hydrophobic residues that sequester $\mathrm{CO}_{2}$ in the active site (28) but does significantly destabilize important hydrophilic regions of the active site containing proton shuttle His 64 as well as $\alpha$-helical regions on the outer surface of the enzyme. 


\section{DISCUSSION}

The catalytic role of the zinc in carbonic anhydrase, in the hydration of $\mathrm{CO}_{2}$, is to lower the $\mathrm{pK}_{\mathrm{a}}$ of the metal bound hydroxide so as to enhance its nucleophilicity as a Lewis acid $(2,5)$. The role of zinc in maintaining the stability of HCA II has been less well studied. Emphasized here is the structural similarity of the apo- to holo-HCA II at atomic resolution, a continuation of the initial study of Håkansson et al. (12). An exception is the conformation of the side chain of His 64, the proton shuttle residue (4) which in the holo-HCA II is equally in an inward and outward orientation that is largely independent of $\mathrm{pH}$ between 6 and $8(6,29)$, whereas in the apo-HCA II His 64 appears predominantly in the outward orientation (Figure 2). This is an interesting result considering that the ordered water in the active-site cavity appears nearly identical in both the apo- and holo-HCA II (Figures 1 and 2) with very similar B factors (Figure 3). Therefore the electrostatic influence of the zinc on the orientation of the side chain of His 64 may play a significant role in establishing its dual orientation in the holo-HCA II. Moreover, this balance between inward and outward orientation of His 64 supports a role for both of these conformations in the proton transfer mechanism (6).

The water molecule, W4 in Figure 2, was observed in a space vacated by the inward orientation of His64 in the apo-HCA II. Although the presence of this additional water is observed only in the apo-HCA II structure, its presence in the holo-HCA II could well be possible. In the catalytically active holo-HCA II, the constant inward and outward flickering of His 64 would render this water a momentary presence that would elude its detection through crystallographic (time averaged) methods. However in circumspect, it should be noted that the possibility of the electron density of W4 to be an artifact (owing to the partial occupancy of the 'in' conformer His 64) cannot be entirely ruled out. Nevertheless if the speculation of W4 is true, it would be interesting to determine if this water molecule plays any significant role in the proton transfer from the zinc- $\mathrm{H}_{2} \mathrm{O}$ to His 64. Furthermore, the captured outward orientation of His 64 has stabilized two additional water molecules, W5 and W6, which allowed their detection through crystallographic methods. These stabilized water molecules in vivo may likely be the first candidates of the bulk solvent to receive protons from the active site during catalysis (Figure 2), although ultimately protons must transfer to the buffer in solvent to maintain the maximum velocity of catalysis.

The observation of nearly identical ordered water structures in apo- and holo- HCA II confirms the role of residues in the active-site cavity in stabilizing this water network, specifically Tyr 7, Asn 62, and Asn 67, among others (27). That is, the zinc plays very little, if any, role in stabilizing the structure of the active-site waters. This study shows there is no significant change in water structure by removing the zinc; however, replacement of Tyr 7, Asn 62, or Asn 67 causes large changes in both the structure of the ordered water and the rate of proton transfer in catalysis (27). Recent work has also established that the zinc in HCA II plays no role in the binding orientation of $\mathrm{CO}_{2}$ in the active-site cavity (28). This is further evidence of the predominant role of the zinc in HCA II, to activate the zinc-bound solvent molecule for nucleophilic addition to $\mathrm{CO}_{2}$.

Clearly the zinc contributes to the thermal stability of the protein. The main unfolding transition is lowered $8{ }^{\circ} \mathrm{C}$ in apo- compared to holo-HCA II (Figures 4, S4 and Table 2). Comparing the crystallographic B factors and backbone amide H/D exchange data to examine the source of this destabilization gave results that were consistent with this observation (Figures 3 and 5). Residues in the vicinity of the zinc were not observably influenced by its removal. Rather, enhanced thermal mobility in the apo-HCA II was observed by B factors and H/D in regions of the enzyme near the surface, especially residues 147-189 containing part of an extended platform of the $\beta$-sheet and an $\alpha$-helix (Figure 5). However it was interesting to note that the enthalpy of unfolding was similar between the apo- and holo-HCA II and the $\Delta \Delta G_{\mathrm{T}=55^{\circ} \mathrm{C}}$ yields 
a small value of $12 \mathrm{kcal} \mathrm{mol}^{-1}$ (Table 2). It maybe possible that the surface residues of a folded enzyme require the attainment of a threshold vibration for the unfolding cascade to be triggered. This threshold seemed to have to been attained at an earlier temperature of the apo-HCA II, possibly because the apo- already possessed a higher thermal vibration in comparison to holoHCA II initially (Figure 3 and S3). Both apo- and holo-HCA II exhibited a two-state cooperative denaturation process. The holo- displayed a sharper peak indicating a higher extent of the cooperative denaturation than the apo-HCA II.

It should also be noted that regions of the active-site cavity associated with catalysis (except His 64) did not change upon removal of zinc. This probably reflects the significance of this region in ordered water formation that maybe critical for proton transfer. That is, mobility in this region would decrease catalysis. The enhanced mobility in apo-HCA II of regions away from the zinc and closer to the surface of the enzyme simply reflect weaker intramolecular interactions in these regions. The source of this effect may again be electrostatic due to removal of the zinc. It may also be due to an angular effect; that is, small mobility near the zinc binding site where there are significant intramolecular interactions may translate to more extensive mobility near the surface of the enzyme where there are fewer such interactions.

\section{Supplementary Material}

Refer to Web version on PubMed Central for supplementary material.

\section{ACKNOWLEDGEMENTS}

We thank Dr. C. K. Tu for insightful discussions. We thank Dr. Joanna R. Long for providing instrumentation for DSC and CD. We also thank Dr. Vijay C. Antharam and R. Suzanne Farver for the help they have rendered with the DSC experimentation and data analysis.

\section{Abbreviations}

CA, carbonic anhydrase; HCA II, human CA isozyme II; Apo-HCA II, HCA II with zinc ion removed; DSC, differential scanning calorimetry; HDX, hydrogen/deuterium exchange; R.m.s.d., root mean square deviation; $\mathrm{T}_{\mathrm{m}}$, melting temperature.

\section{REFERENCES}

1. Krishnamurthy VM, Kaufman GK, Urbach AR, Gitlin I, Gudiksen KL, Weibel DB, Whitesides GM. Carbonic anhydrase as a model for biophysical and physical-organic studies of proteins and protein ligand binding. Chem Rev 2008;108:946-1051. [PubMed: 18335973]

2. Lindskog S. Structure and mechanism of carbonic anhydrase. Pharmacol. Ther 1997;74:1-20. [PubMed: 9336012]

3. Silverman DN, Lindskog S. The catalytic mechanism of carbonic anhydrase: implications of a ratelimiting protolysis of water. Acc. Chem. Res 1988;21:30-36.

4. Tu CK, Silverman DN, Forsman C, Jonsson BH, Lindskog S. Role of histidine 64 in the catalytic mechanism of human carbonic anhydrase II studied with a site-specific mutant. Biochemistry 1989;28:7913-7918. [PubMed: 2514797]

5. Christianson DW, Fierke CA. Carbonic anhydrase: evolution of the zinc binding site by nature and by design. Acc. Chem. Res 1996;29:331-339.

6. Fisher SZ, Maupin CM, Budayova-Spano M, Govindasamy L, Tu CK, Agbandje-McKenna M, Silverman DN, Voth GA, McKenna R. Atomic Crystal and Molecular Dynamics Simulation Structures of Human Carbonic Anhydrase II: Insights into the Proton Transfer Mechanism. Biochemistry 2007;42:2930-2937. [PubMed: 17319692] 
7. Liljas A, Kannan KK, Bergstén PC, Waara I, Fridborg K, Strandberg B, Carlbom U, Järup L, Lövgren S, Petef M. Crystal structure of human carbonic anhydrase c. Nat New Biol 1972;235(57):131-137. [PubMed: 4621826]

8. Cox JD, Hunt JA, Compher KM, Fierke CA, Christianson DW. Structural influence of hydrophobic core residues on metal binding and specificity in carbonic anhydrase II. Biochemistry 2000;39:1368713694. [PubMed: 11076507]

9. McCall KA, Fierke CA. Probing determinants of the metal ion selectivity in carbonic anhydrase using mutagenesis. Biochemistry 2004;43:3979-3986. [PubMed: 15049705]

10. Lindskog S. Effects of $\mathrm{pH}$ and inhibitors on some properties related to metal binding in bovine carbonic anhydrase. J. Biol. Chem 1963;238:945-951. [PubMed: 13930837]

11. Bertini I, Luchinat C, Scozzafava A. Carbonic anhydrase - an insight into the zinc-binding site and into the active cavity through metal substitution. Structure and Bonding 1982;48:45-92.

12. Hakansson K, Carlsson M, Svensson LA, Liljas A. Structure of native and apo carbonic anhydrase II and structure of some of its anion-ligand complexes. J. Mol. Biol 1992;227:1192-1204. [PubMed: 1433293]

13. Forsman CA, Behravan G, Osterman A, Jonsson BH. Production of active human carbonic anhydrase II in E. coli. Acta. Chem. Scand 1988;42:314-318.

14. Khalifah RG, Strader DJ, Bryant SH, Gibson SM. Carbon-13 nuclear magnetic resonance probe of active site ionizations in human carbonic anhydrase. Biochemistry 1977;16:2241-2247. [PubMed: 16641]

15. Silverman DN. Carbonic anhydrase: oxygen-18 exchange catalyzed by an enzyme with ratecontributing proton-transfer steps. Methods Enzymol 1982;87:732-752. [PubMed: 6294458]

16. McPherson, A. Preparation and Analysis of Protein Crystals. Vol. 1st Ed.. Wiley; New York: 1982.

17. Otwinowski Z, Minor W. Processing of x-ray diffraction data collected in oscillation mode. Methods Enzymol 1997;276:307-326.

18. Sheldrick GM. A Short History of SHELX. Acta Crystallogr. Sect. A 2008;64:112-122. [PubMed: 18156677]

19. Brunger AT. Free R value: A novel statistical quantity for assessing the accuracy of crystal structures. Nature 1992;355:472-475. [PubMed: 18481394]

20. Emsley P, Cowtan K. Coot: model-building tools for molecular graphics. Acta Crystallogr. Sect. D 2004;60:2126-2132. [PubMed: 15572765]

21. Laskowski RA, MacArthur MW, Moss DS, Thornton JM. PROCHECK: a program to check the stereochemical quality of protein structures. J. Appl. Cryst 1993;26:283-291.

22. Chalmers MJ, Busby SA, Pascal BD, He Y, Hendrickson CL, Marshall AG, Griffin PR. Probing protein ligand interactions by automated hydrogen/deuterium exchange mass spectrometry. Anal. Chem 2006;78(4):1005-1014. [PubMed: 16478090]15

23. Chalmers MJ, Busby SA, Pascal BD, Southern MR, Griffin PR. A two-stage differential hydrogen deuterium exchange method for the rapid characterization of protein/ligand interactions. J. Biomol. Tech 2007;18(4):194-204. [PubMed: 17916792]

24. Pascal BD, Chalmers MJ, Busby SA, Mader CC, Southern MR, Tsinoremas NF, Griffin PR. The deuterator: software for the determination of backbone amide deuterium levels from H/D exchange MS data. BMC Bioinformatics 2007;8:156. [PubMed: 17506883]

25. Pascal BD, Chalmers MJ, Busby SA, Griffin PR. HD desktop: an integrated platform for the analysis and visualization of H/D exchange data. J. Am. Soc. Mass Spectrom 2009;20(4):601-610. [PubMed: 19135386]

26. Becktel WJ, Schellman JA. Biopolymers 1987;26:1859-1877. [PubMed: 3689874]

27. Fisher SZ, Tu CK, Bhatt D, Govindasamy L, Agbandje-McKenna M, McKenna R, Silverman DN. Speeding up proton transfer in a fast enzyme: kinetic and crystallographic studies on the effect of hydrophobic amino acid substitution in the active site of human carbonic anhydrase II. Biochemistry 2007;42:3803-3813. [PubMed: 17330962]

28. Domsic JF, Avvaru BS, Kim CU, Gruner SM, Agbandje-McKenna M, Silverman DN, Mckenna R. Entrapment of Carbon Dioxide in the active site of carbonic Anhydrase II. J. Biol. Chem 2008;283:30766-30771. [PubMed: 18768466] 
29. Nair SK, Christianson DW. Unexpected pH-Dependent Conformation of His-64, the Proton Shuttle of Carbonic Anhydrase-II. J. Am. Chem. Soc 1991;113:9455-9458. 


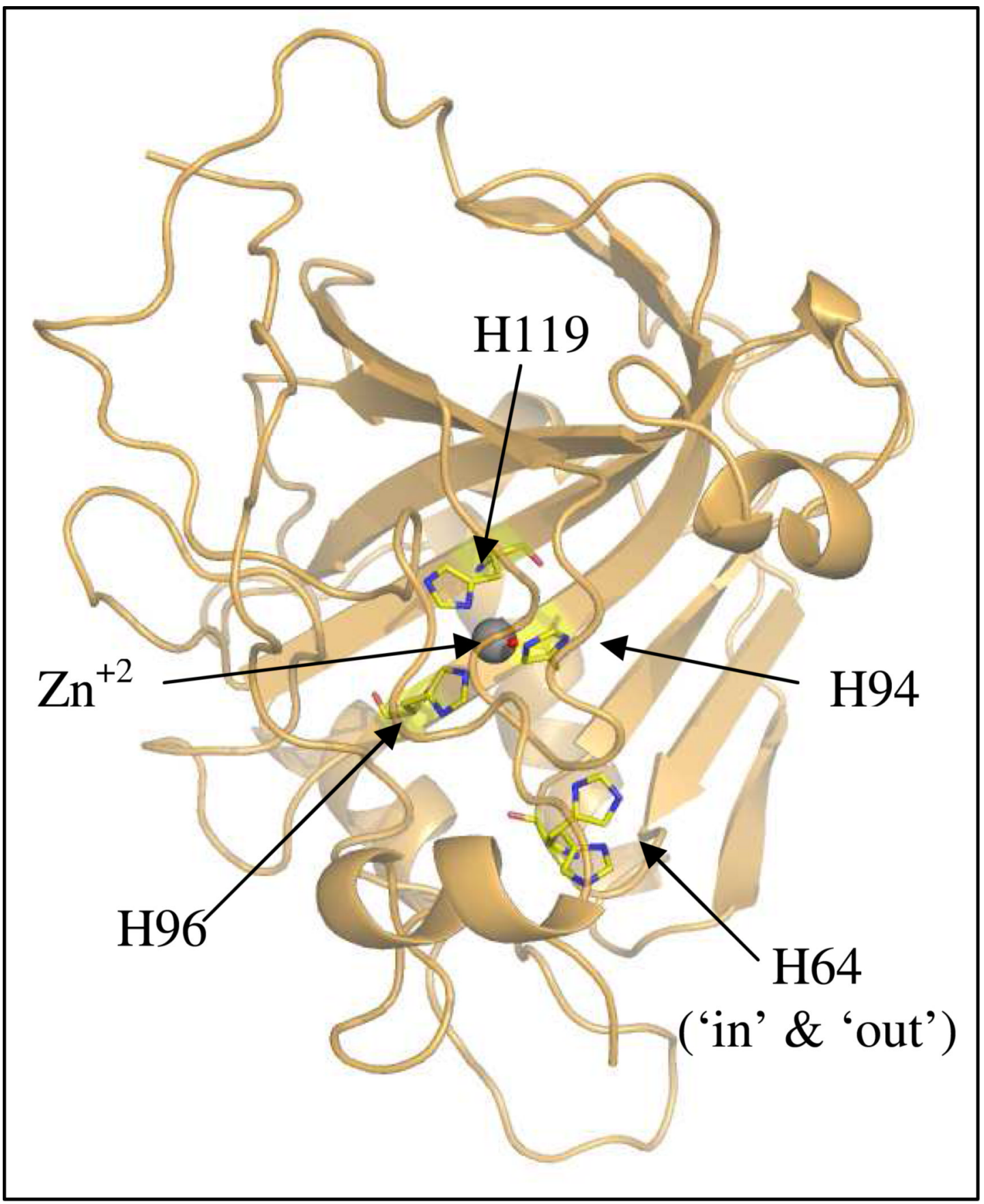

Figure 1.

Overall structure of holo-HCA II. Cartoon depiction of the secondary structural elements are as shown. Structure used was (PDB ID: 2ILI, (2)). Zinc atom represented as a grey sphere, histidine residues His 94, His 96, His 119, and the dual conformation of His 64 are represented in stick model representation (carbon, yellow; oxygen, red; nitrogen, blue). Figure made using PyMOL (DeLano Scientific). 


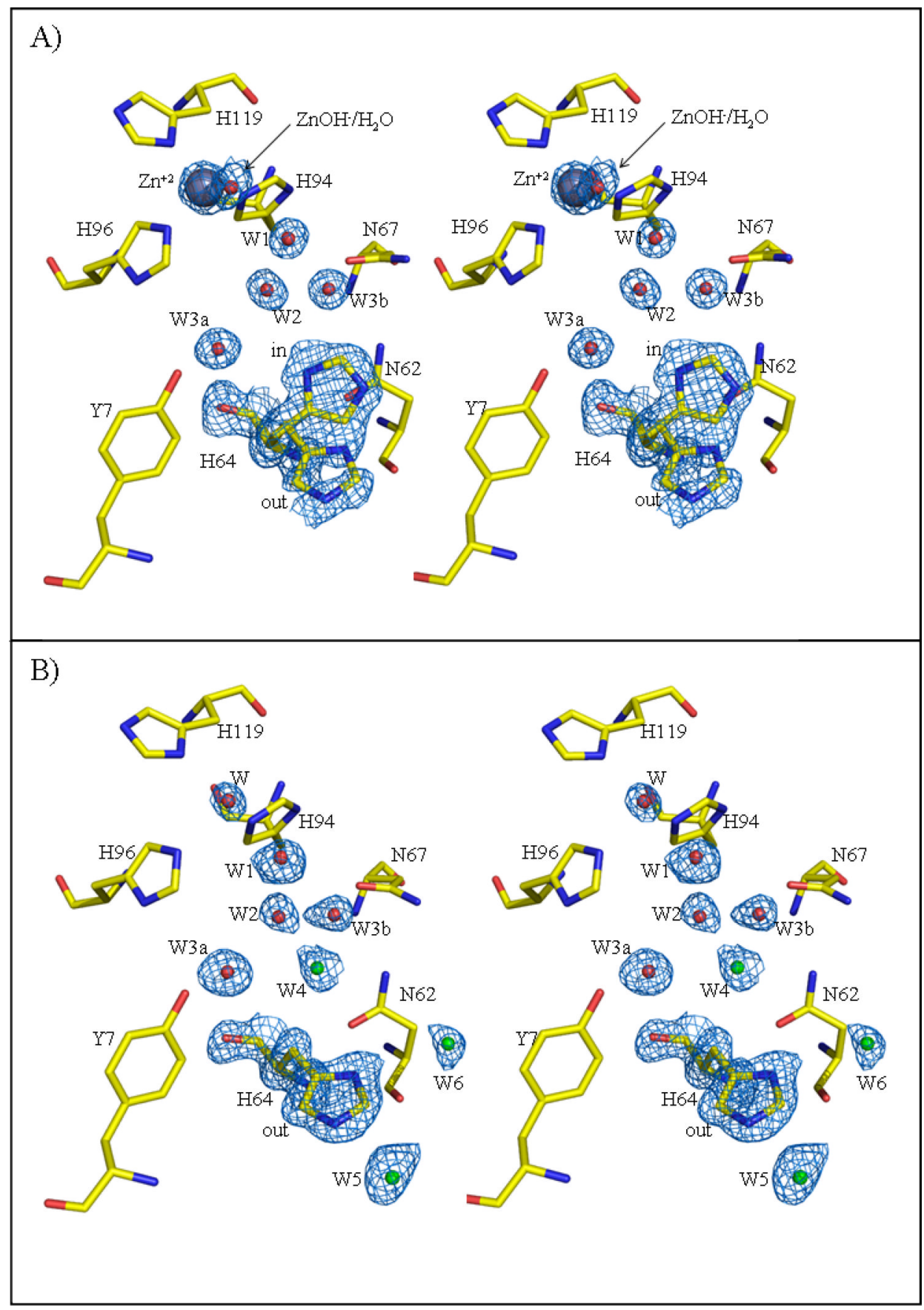

Figure 2.

Active site of holo- and apo-HCA II. Stereoview of the active site of (A) holo- (PDB ID: 2ILI, (2)) and (B) apo-HCA II (this study). The amino acids are as labeled and represented in stick form (carbon, yellow; oxygen, red; nitrogen, blue). The electron density for both the holo- and apo-HCA II is represented by a blue mesh $1.5 \sigma$-weighted $2 F o-F c$ Fourier map. The "common" waters are colored red and the additional waters in the apo-HCA II (W4, W5, and W6) are colored green. Figures are made using PyMOL (DeLano Scientific). 


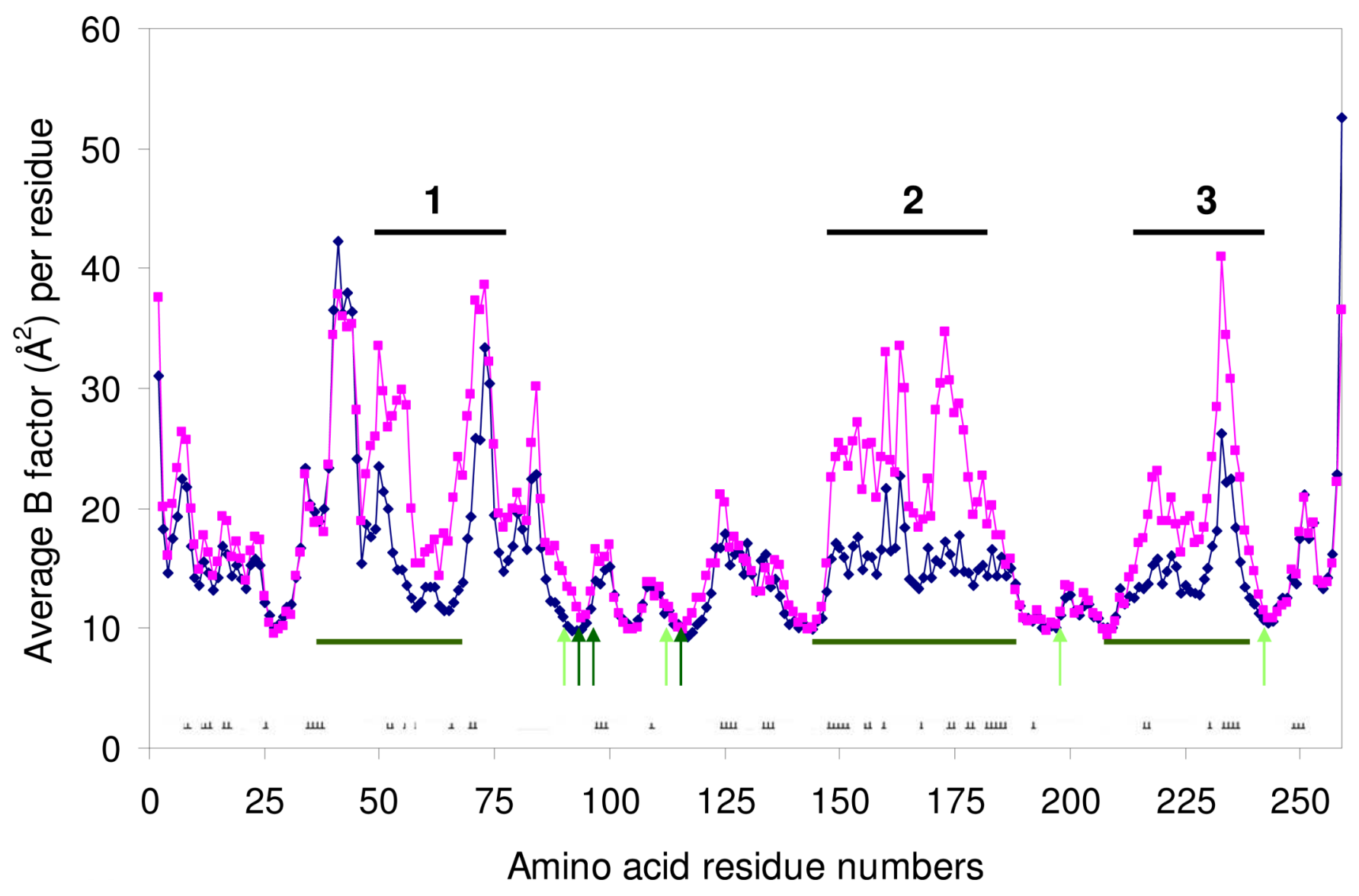

Figure 3.

Plot of the average residue B factors of holo- (dark blue) and apo-HCA II (pink). Regions labeled 1, 2 and 3 (indicated by the black bars) are segments of apo- that have higher B factors than holo-HCA II. The green bars at the base of the figure indicate regions that have the largest extent of difference (up to 12\%) in H/D exchange rates between apo- and holo-HCA II. The dark green arrows indicate first shell ligands, whilst light green arrows indicate second shell ligands of the zinc in holo-HCA II. The tick marks above the abscissa represents regions of crystal contacts within the crystal lattice. 
A)

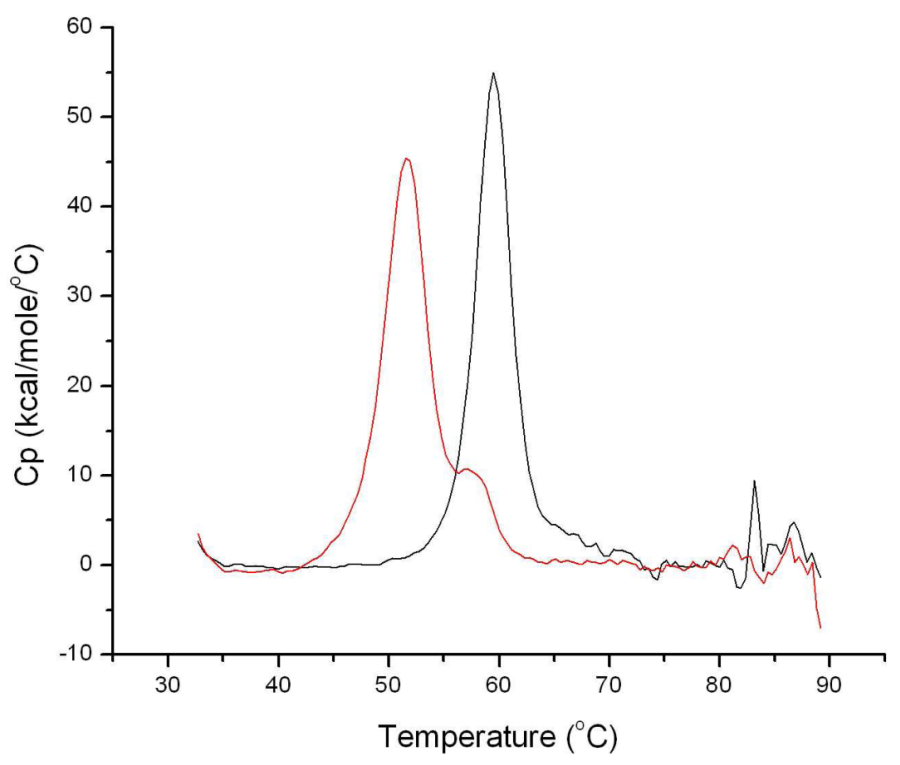

B)

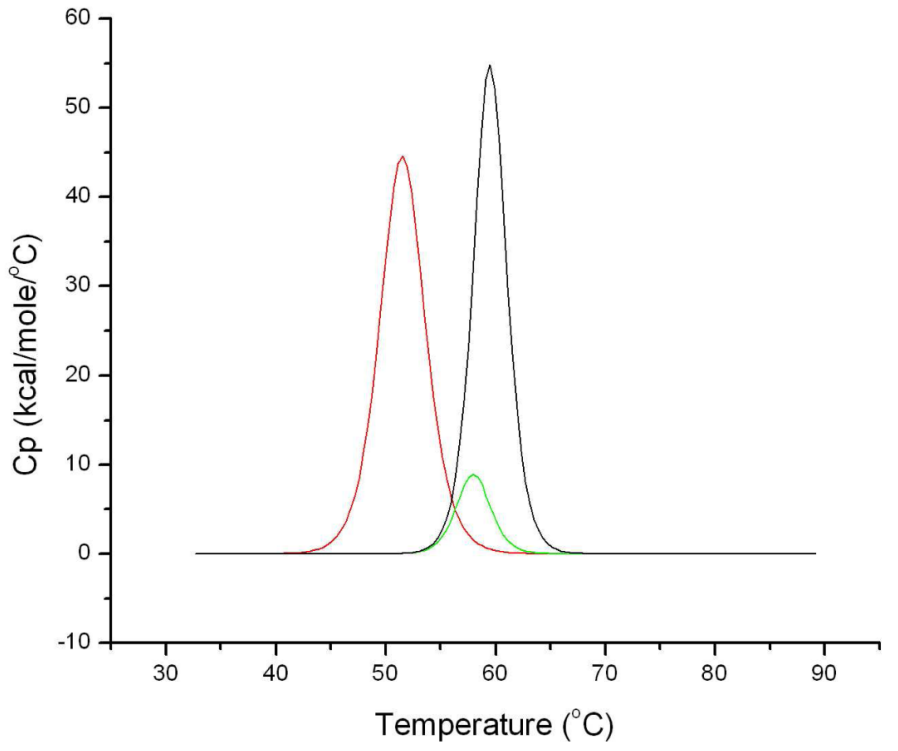

Figure 4.

Differential scanning calorimetry of holo- and apo-HCA II. (A) DSC scans from 30 to $90{ }^{\circ} \mathrm{C}$ of holo- (black) and apo-HCA II (red), shown the dominant transition peaks with values of $\mathrm{T}_{\mathrm{m}}$ of 59 and $51{ }^{\circ} \mathrm{C}$ respectively. (B) The DSC curve of apo-HCA II from panel (A) was resolved into two independent peaks red and green. The secondary peak (green) coincides with holo-HCA II peak (black) and is attributed to 10\% holo-HCA II contamination within apoHCA II samples (see text for explanation). 
A)

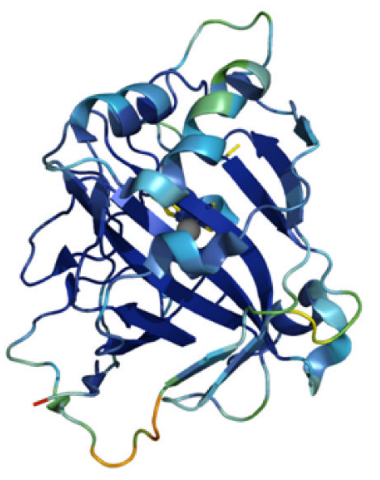

B)

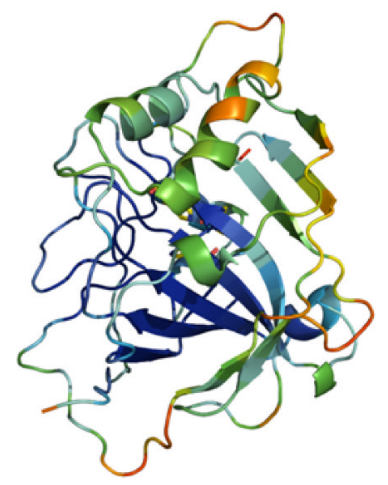

C)

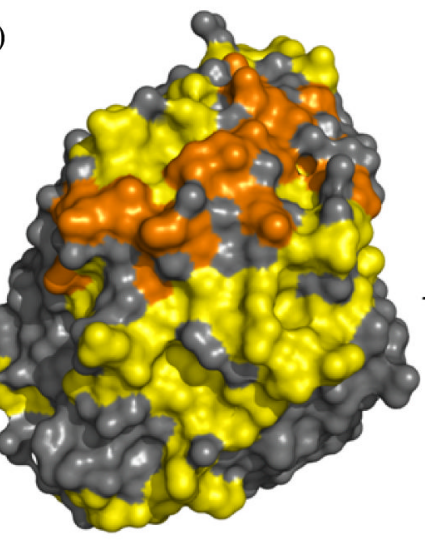

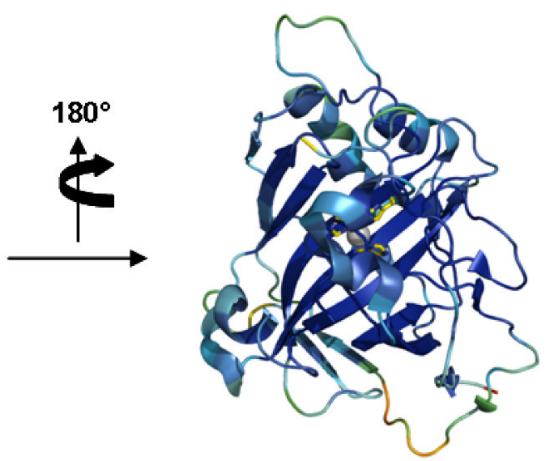
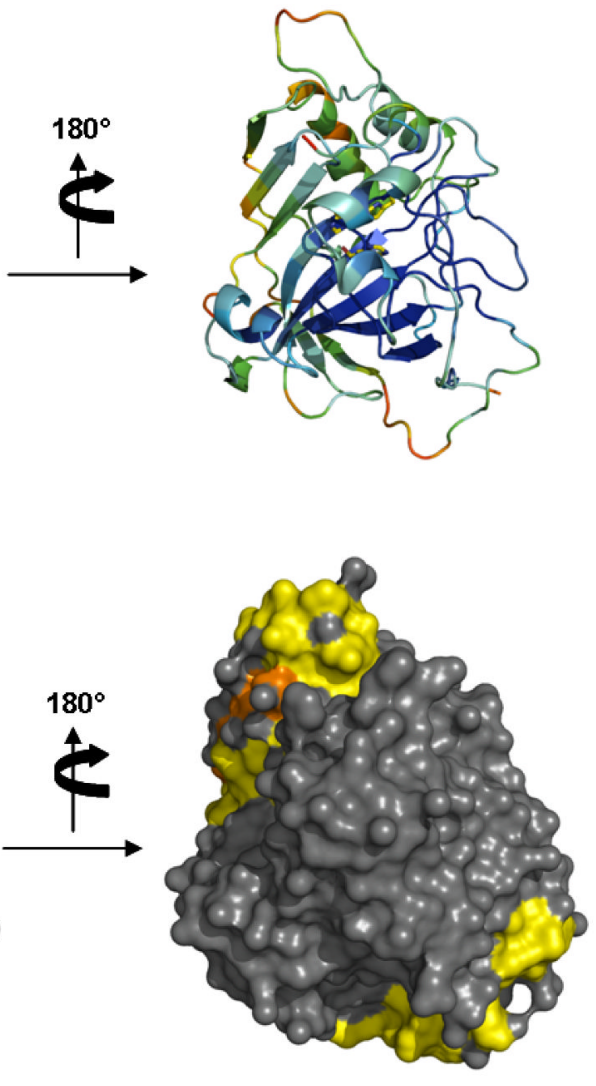

Figure 5.

Cartoon and surface renditions of crystallographic B factors and differential H/D exchange of holo- and apo-HCA II. Cartoon (A) holo- and (B) apo-HCA II color coded based on their respective normalized $\mathrm{B}$ factors. The color transition from blue to red depicts the relative increase of B factor from the lowest to highest (see Figure 3). (C) Surface profile comparing backbone amide exchange rates of apo-HCA II to holo-HCA II overlaid onto the apo-HCA II crystal structure. Differences in deuterium incorporation for each of 36 regions of HCA II (see Table S2 and Figure S1) were determined by subtracting the mean exchange rates for each region of the apo-HCA II from the mean exchange rates of the same regions of the holo-HCA II for the exchange times of 1, 30, 60, 900, 3600 and $260000 \mathrm{~s}$. Regions colored yellow represent 
increases of $\mathrm{H} / \mathrm{D}$ amide exchange rates between 5-9\% and regions colored orange represent increases of H/D amide exchange rates between 10-20\% of apo-HCA II relative to holo-HCA II. Regions colored gray represent areas of non-significant changes in H/D amide exchange rates between the two forms of the enzyme and regions colored white could not be experimentally measured. Figures made using PyMOL (Delano Scientific). 
Table 1

Refinement and Final Model Statistics for the Crystallographic Study of Apo-HCA II

\begin{tabular}{|c|c|}
\hline \multicolumn{2}{|l|}{ Data-collection statistics } \\
\hline Temperature (K) & 100 \\
\hline Wavelength $(\AA)$ & 0.9772 \\
\hline$\stackrel{\text { Space group }}{\stackrel{c}{\rightleftharpoons}}$ & $\mathrm{P} 2_{1}$ \\
\hline Unit-cell parameters $\left(\AA{ }^{\circ}{ }^{\circ}\right)$ & $a=42.7, b=41.6 c=72.8, \beta=104.5$ \\
\hline \multirow{2}{*}{$\begin{array}{l}\text { Total number of reflections } \\
\text { Total number of unique reflections }\end{array}$} & $476977(15426)^{*}$ \\
\hline & $61768(5933)^{*}$ \\
\hline Resolution $(\AA)$ & $50-1.26(1.31-1.26)^{*}$ \\
\hline $\mathrm{R}_{\text {sym }}$ & $0.057(0.194)^{*}$ \\
\hline $\mathrm{I} / \sigma(\mathrm{I})$ & $29.7(10.0)^{*}$ \\
\hline$a_{\mathrm{R}_{\text {cryst }}(\%)}$ & 14.0 \\
\hline$b_{\mathrm{R}_{\text {free }}(\%)}$ & 18.7 \\
\hline Residue Nos. & $4-261$ \\
\hline No. of protein atoms & 2087 \\
\hline No. of $\mathrm{H}_{2} \mathrm{O}$ molecules & 250 \\
\hline R.m.s.d. for bond lengths $(\AA)$, angles $\left(^{\circ}\right)$ & $0.004,1.4$ \\
\hline \multicolumn{2}{|l|}{ Ramachandran statistics (\%) } \\
\hline Most favored, additionally allowed and generously allowed regions & $88,11.5,0.5$ \\
\hline \multicolumn{2}{|l|}{ B factors $\left(\AA^{2}\right)$} \\
\hline Average, main-, side-chain, solvent & $18.6,23.1,30.2$ \\
\hline \multicolumn{2}{|l|}{${ }^{b} \mathrm{R}_{\text {cryst }}=\left(\Sigma|F \mathrm{o}|-|F \mathrm{c}| / \Sigma\left|F_{\text {obs }}\right|\right) \times 100}$. \\
\hline${ }^{c} \mathrm{R}_{\text {free }}$ is calculated in same manner as ${ }^{b_{\mathrm{R}}}$ cryst, except that it uses $5 \%$ of the reflection data omitted from refinement. & ion data omitted from refinement. \\
\hline
\end{tabular}




\section{Table 2}

Thermodynamics of Unfolding of Apo- and Holo-HCA II

\begin{tabular}{|c|c|c|c|}
\hline Parameter & Apo-HCA II & Holo-HCA II & \\
\hline$T_{\mathrm{m}}\left({ }^{\circ} \mathrm{C}\right)^{a}$ & $51 \pm 0.5$ & $59 \pm 0.5$ & \\
\hline$\Delta H^{\circ} \mathrm{m}\left(\mathrm{kcal} \mathrm{mol}^{-1}\right)^{a}$ & $280 \pm 20$ & $250 \pm 20$ & \\
\hline$\Delta H^{\mathrm{vH}}\left(\mathrm{kcal} \mathrm{mol}^{-1}\right)^{b}$ & $200 \pm 20$ & $220 \pm 20$ & \\
\hline$\Delta C_{p}\left(\mathrm{kcal} \mathrm{mol}^{-1} \mathrm{~K}^{-1}\right)^{c}$ & 0.86 & 0.75 & \\
\hline$\Delta G_{\mathrm{T}=55^{\circ} \mathrm{C}}\left(\mathrm{kcal} \mathrm{mol}^{-1}\right)^{d}$ & -9.3 & +3.0 & \\
\hline$\Delta H_{\mathrm{T}=55^{\circ} \mathrm{C}}\left(\mathrm{kcal} \mathrm{mol}^{-1}\right)^{e}$ & 283 & 247 & \\
\hline$\Delta S_{\mathrm{T}=55^{\circ} \mathrm{C}}\left(\mathrm{kcal} \mathrm{mol}^{-1}\right)^{f}$ & 0.87 & 0.74 & \\
\hline \multicolumn{4}{|c|}{${ }^{a}$ Calorimetric parameters determined by DSC. } \\
\hline \multicolumn{4}{|c|}{ 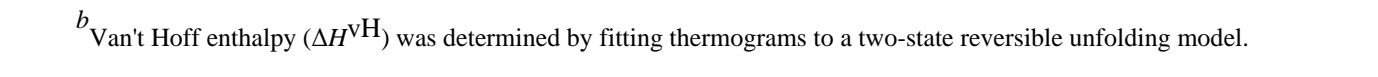 } \\
\hline \multicolumn{4}{|c|}{${ }^{c}$ Heat capacity $\left(\Delta C_{p}\right)$ of protein unfolding obtained by plotting calorimetric enthalphy $\left(\Delta H^{\circ} \mathrm{m}\right)$ vs melting temperature $\left(T_{\mathrm{m}}\right)$. } \\
\hline \multicolumn{4}{|c|}{ 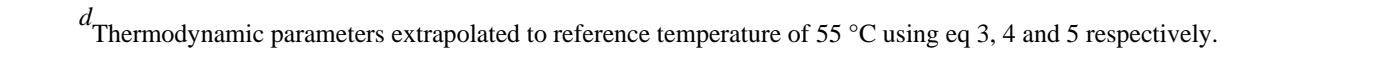 } \\
\hline \multicolumn{4}{|c|}{$e^{e}$ Thermodynamic parameters extrapolated to reference temperature of $55^{\circ} \mathrm{C}$ using eq 3,4 and 5 respectively. } \\
\hline \multicolumn{4}{|c|}{ 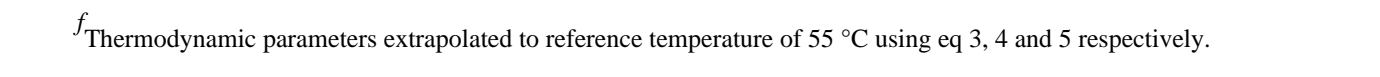 } \\
\hline
\end{tabular}

